Article

\title{
Preliminary Estimation of Deoxynivalenol Excretion through a 24 h Pilot Study
}

\section{Yelko Rodríguez-Carrasco ${ }^{\dagger}$, Jordi Mañes, Houda Berrada ${ }^{\dagger} * *$ and Guillermina Font}

Area of Toxicology, Department of Public Health, Faculty of Pharmacy, University of Valencia, Burjassot 46100, Spain; E-Mails: yelko.rodriguez@uv.es (Y.R.-C.); jordi.manes@uv.es (J.M.); guillermina.font@uv.es (G.F.)

$\dagger$ These authors contributed equally to this work.

* Author to whom correspondence should be addressed; E-Mail: houda.berrada@uv.es; Tel.: +34-96-354-4117 (ext. 44117); Fax: +34-96-354-4954.

Academic Editor: Sven Dänicke

Received: 16 December 2014 / Accepted: 16 February 2015 / Published: 25 February 2015

\begin{abstract}
A duplicate diet study was designed to explore the occurrence of 15 Fusarium mycotoxins in the $24 \mathrm{~h}$-diet consumed by one volunteer as well as the levels of mycotoxins in his $24 \mathrm{~h}$-collected urine. The employed methodology involved solvent extraction at high ionic strength followed by dispersive solid phase extraction and gas chromatography determination coupled to mass spectrometry in tandem. Satisfactory results in method performance were achieved. The method's accuracy was in a range of $68 \%-108 \%$, with intra-day relative standard deviation and inter-day relative standard deviation lower than $12 \%$ and $15 \%$, respectively. The limits of quantitation ranged from 0.1 to $8 \mu \mathrm{g} / \mathrm{Kg}$. The matrix effect was evaluated and matrix-matched calibrations were used for quantitation. Only deoxynivalenol (DON) was quantified in both food and urine samples. A total DON daily intake amounted to $49.2 \pm 5.6 \mu \mathrm{g}$ whereas DON daily excretion of $35.2 \pm 4.3 \mu \mathrm{g}$ was determined. DON daily intake represented $68.3 \%$ of the established DON provisional maximum tolerable daily intake (PMTDI). Valuable preliminary information was obtained as regards DON excretion and needs to be confirmed in large-scale monitoring studies.
\end{abstract}

Keywords: deoxynivalenol; duplicate diet study; GC-MS/MS; urine; risk characterization 


\section{Introduction}

Cereals are the most important source of food for both direct human consumption and livestock production. In fact, the latest published data by the Food and Agricultural Organization (FAO) reported an annually cereal global consumption (excluding beer) of $146.7 \mathrm{~kg} / \mathrm{capita}$ [1]. Mycotoxins are secondary fungi metabolites produced in several commodities that could exert toxic effects on animals and humans [2] and mycotoxin contamination of cereals is also frequently reported as a public health threat $[3,4]$. Acute exposures to mycotoxins are related to gastrointestinal manifestations such as diarrhea, vomiting and melena, while chronic exposures and the most worrisome one are related to degenerative effects on the immune, neural and reproductive systems. Chronic exposure to some mycotoxins are also related to cancer induction [5]. Mycotoxins have also been classified as priority food contaminants by the Global Environment Monitoring System-Food Contamination Monitoring Assessment Programme (GEMS/Food) of the World Health Organization (WHO) [6]. Moreover, mycotoxins were the main hazards in the European Union with 425 border rejection notifications as highlighted its annual report for 2012, according to the Rapid Alert System for Food and Feed [7].

Among the wide number of mycotoxins, zearalonene and trichothecenes (type A mainly represented by T-2 and HT-2 toxins, and type B by deoxynivalenol (DON)) constitute one of the largest groups of mycotoxins produced by Fusarium in cereal grains [8,9]. Mycotoxin production in agricultural crops can occur at various stages in the food chain like pre-harvest, harvest, drying and storage. In addition, mycotoxins tend to persist during the transformation and processing of contaminated plants and are also still reported in cooked and sterilized food [10].

The European Commission (EC) has set maximum limits for some mycotoxins in foodstuffs [11,12] and the Joint FAO/WHO Expert Committee on Food Additives (JEFCA) established maximum tolerable daily intakes to ensure food safety [13]. According to the guidelines published by the WHO, the basic approaches that can be used to determine the intake of a food contaminant include: total diet studies, duplicate diet studies, and selective studies of individual foods, which combine food consumption patterns and contamination level [14]. Duplicate diet studies may be a good alternative to total diet studies, especially when there are important economical limitations to perform a suitable total diet studies. In addition, duplicate diet studies are particularly interesting to consider not only basic cooking methods, but real cooking, something essential when evaluating the dietary intake in specific individuals, countries or regions [15]. A step forward in the individual exposure assessment could be provided by biomarkers measured in biological fluids. The identification of mycotoxins and their main metabolized products in urine could therefore serve as such biomarkers and could facilitate effective exposure assessment $[16,17]$.

Knowledge of mycotoxins in vivo metabolism in humans has been rarely investigated and it is fundamental to carry out some studies which serve as an approach to assess the exposure. In this line, some experiments were designed to provide tentative information about the human in vivo metabolism of major Fusarium mycotoxins. For instance, Mirocha et al. [18] studied the zearalenone metabolite pattern in $24 \mathrm{~h}$ urine after ingestion of $100 \mathrm{mg}$ zearalenone at once by one volunteer. Similarly, Warth et al. [19] carried out a study to investigate the human deoxynivalenol and zearalenone in vivo metabolism through the analysis of urine samples obtained from one volunteer following a naturally contaminated diet containing $138 \mu \mathrm{g}$ DON and $10 \mu \mathrm{g}$ ZON over a period of four days. Thus, this 
preliminary work was designed as the previously ones to provide basic information about the human in vivo metabolism and serves for the purpose to develop a method which might principally suited as a screening tool. The dietary intake of 15 mycotoxins was studied in a single individual using the duplicate diet approach. All food products as prepared, served and consumed were analyzed. The occurrence of mycotoxins and metabolites was also evaluated in the 24-hour urine collection and expressed by $\mu \mathrm{g} / \mathrm{g}$ of creatinine. The aims of the study were to investigate the daily mycotoxin intake through complete cooked meals, to estimate the mycotoxin urinary excretion, and to carry out a risk characterization approach for the participant.

\section{Results and Discussion}

\subsection{Method Performance}

Regression equations were obtained using eight standard concentrations on the abscissa and the area of the chromatogram peaks as vertical coordinates. Linear range was tested at eight concentration levels in triplicate from LOQ to $100 \mu \mathrm{g} / \mathrm{kg}$. Relative standard deviations among the triplicate were below $5 \%$ at all calibration curve points. The determination coefficients $\left(R^{2}\right)$ of all analytes were $>0.995$. The matrix effect was observed (from $83 \%$ to $91 \%$ ), and thus matrix-matched calibration curves were used for quantification purposes. Apparent recovery for each mycotoxin was determined in composite, beer and urine samples spiked at low and high level (Table 1).

Recoveries values obtained, from 68 to $108 \%$, were in agreement with the range set in legislation [20]. Corrections based on recovery percentages were not performed. A precision study was performed by determining the repeatability (intra-day precision) $(n=6)$ and reproducibility (inter-day precision) $(n=4)$, and was fulfilled in conformity with the described criteria in current legislation. Intra-day and inter-day precision were lower than 12 and 15\%, respectively in the assayed matrices. The sensitivity of the method was expressed in terms of limits of detection (LOD) and limits of quantitation (LOQ). LOD and LOQ values were calculated from spiked samples chromatograms based on a signal to noise ratio of $3: 1$ and 10:1, respectively (Table 1). LODs varied in the following ranges of $0.6-5 \mu \mathrm{g} / \mathrm{kg}, 0.05-8 \mu \mathrm{g} / \mathrm{kg}$ and $0.1-4 \mu \mathrm{g} / \mathrm{kg}$ for composite, beer and urine respectively. LOQs varied in the following ranges of $1.2-10 \mu \mathrm{g} / \mathrm{kg}, \quad 0.1-16 \mu \mathrm{g} / \mathrm{kg}$ and $0.2-8 \mu \mathrm{g} / \mathrm{kg}$, for composite, beer and urine respectively, which guaranteed quantitation at low ppb-level. 
Table 1. Method performance for fifteen mycotoxins and metabolites in composite diet, beer and urine.

\begin{tabular}{|c|c|c|c|c|c|c|c|c|c|c|c|c|}
\hline \multirow{3}{*}{ Analyte } & \multicolumn{4}{|c|}{ Composite diet } & \multicolumn{4}{|c|}{ Beer } & \multicolumn{4}{|c|}{ Urine } \\
\hline & \multicolumn{2}{|c|}{$\operatorname{REC} \pm \operatorname{RSD}(\%)$} & \multirow{2}{*}{$\begin{array}{c}\text { LOD } \\
(\mu \mathrm{g} / \mathrm{kg})\end{array}$} & \multirow{2}{*}{$\begin{array}{c}\text { LOQ } \\
(\mu \mathrm{g} / \mathrm{kg})\end{array}$} & \multicolumn{2}{|c|}{$\operatorname{REC} \pm \operatorname{RSD}(\%)$} & \multirow{2}{*}{$\begin{array}{c}\text { LOD } \\
(\mu \mathrm{g} / \mathrm{kg})\end{array}$} & \multirow{2}{*}{$\begin{array}{c}\text { LOQ } \\
(\mu \mathrm{g} / \mathrm{kg})\end{array}$} & \multicolumn{2}{|c|}{$\operatorname{REC} \pm \operatorname{RSD}(\%)$} & \multirow{2}{*}{$\begin{array}{c}\text { LOD } \\
(\mu \mathrm{g} / \mathrm{kg})\end{array}$} & \multirow{2}{*}{$\begin{array}{c}\mathrm{LOQ} \\
(\mu \mathrm{g} / \mathrm{kg})\end{array}$} \\
\hline & Low level ${ }^{a}$ & High level $^{\mathrm{c}}$ & & & Low level $^{\mathbf{b}}$ & High level $^{c}$ & & & Low level $^{\text {a }}$ & High level $^{\mathrm{c}}$ & & \\
\hline DOM-1 & $87 \pm 7$ & $93 \pm 5$ & 0.6 & 1.2 & $73 \pm 6$ & $77 \pm 8$ & 0.1 & 0.2 & $84 \pm 2$ & $86 \pm 4$ & 0.2 & 0.5 \\
\hline DON & $89 \pm 5$ & $91 \pm 6$ & 0.6 & 1.2 & $75 \pm 9$ & $83 \pm 9$ & 0.05 & 0.1 & $96 \pm 4$ & $94 \pm 8$ & 0.1 & 0.2 \\
\hline 3-ADON & $95 \pm 4$ & $90 \pm 7$ & 0.6 & 1.2 & $82 \pm 6$ & $80 \pm 5$ & 2 & 4 & $92 \pm 5$ & $94 \pm 5$ & 0.2 & 0.5 \\
\hline FUS-X & $84 \pm 5$ & $89 \pm 3$ & 2.5 & 5 & $98 \pm 8$ & $93 \pm 9$ & 8 & 16 & $95 \pm 3$ & $90 \pm 6$ & 2 & 4 \\
\hline DAS & $103 \pm 3$ & $99 \pm 6$ & 2.5 & 5 & $78 \pm 6$ & $82 \pm 5$ & 4 & 8 & $89 \pm 4$ & $84 \pm 8$ & 1 & 2 \\
\hline NIV & $79 \pm 6$ & $82 \pm 5$ & 1.2 & 2.5 & $77 \pm 12$ & $81 \pm 9$ & 0.5 & 1 & $87 \pm 3$ & $93 \pm 6$ & 0.5 & 1 \\
\hline NEO & $97 \pm 8$ & $92 \pm 5$ & 2.5 & 5 & $83 \pm 8$ & $88 \pm 6$ & 2 & 4 & $93 \pm 5$ & $94 \pm 5$ & 0.2 & 0.5 \\
\hline HT-2 & $93 \pm 7$ & $89 \pm 8$ & 1.2 & 2.5 & $97 \pm 9$ & $93 \pm 4$ & 2 & 4 & $96 \pm 4$ & $91 \pm 8$ & 1 & 2 \\
\hline $\mathrm{T}-2$ & $84 \pm 9$ & $90 \pm 6$ & 2.5 & 5 & $108 \pm 7$ & $97 \pm 8$ & 4 & 8 & $102 \pm 6$ & $94 \pm 9$ & 0.5 & 1 \\
\hline ZAN & $85 \pm 5$ & $90 \pm 4$ & 5 & 10 & $68 \pm 9$ & $73 \pm 9$ & 8 & 16 & $72 \pm 7$ & $74 \pm 8$ & 4 & 8 \\
\hline$\alpha-Z A L$ & $72 \pm 8$ & $79 \pm 8$ & 5 & 10 & $70 \pm 6$ & $78 \pm 7$ & 4 & 8 & $79 \pm 5$ & $82 \pm 5$ & 4 & 8 \\
\hline$\beta-Z A L$ & $79 \pm 6$ & $77 \pm 6$ & 5 & 10 & $73 \pm 8$ & $79 \pm 8$ & 4 & 8 & $77 \pm 8$ & $74 \pm 6$ & 4 & 8 \\
\hline $\mathrm{ZON}$ & $87 \pm 8$ & $84 \pm 7$ & 2.5 & 5 & $71 \pm 5$ & $78 \pm 6$ & 8 & 16 & $81 \pm 5$ & $84 \pm 7$ & 3 & 6 \\
\hline$\alpha-\mathrm{ZOL}$ & $83 \pm 9$ & $80 \pm 7$ & 2.5 & 5 & $78 \pm 6$ & $83 \pm 4$ & 2 & 4 & $88 \pm 2$ & $93 \pm 5$ & 1 & 2 \\
\hline$\beta-\mathrm{ZOL}$ & $77 \pm 6$ & $78 \pm 9$ & 2.5 & 5 & $74 \pm 8$ & $73 \pm 8$ & 4 & 8 & $80 \pm 6$ & $84 \pm 9$ & 2 & 4 \\
\hline
\end{tabular}




\subsection{Deoxynivalenol Reduction during Cooking}

Mycotoxin analyses of pasta (spaghetti) and whole-wheat pasta (little stars) were carried out prior the cooking step and then cooked after the drying process. Analyses were performed in triplicate. The purpose was to evaluate the percentage of mycotoxin reduction during food preparation procedure. Most of the exposure assessment approach to contaminants has been carried out based on un-cooked food, and thus assuming some uncertainty in the reported data. In this work, not only cooked meals but also the regular cooking practices and the serving size were taking into account. The aim was not to serve as a representative data of percentage of reduction for wheat-based products, but to minimize the uncertainty of the obtained data in order to allow a closer exposure assessment approach. A reduction of $13 \%$ and $58 \%$ was obtained for whole-wheat pasta and pasta, respectively after culinary treatment (Figure 1). That difference could be attributed to the distinct serving. While the cooking water is removed in spaghetti serving, little stars are consumed as a soup and thus, the amount of mycotoxin intake in little stars was higher than in spaghetti. The percentage of reduction obtained in this work were comparable than those reported Visconti et al. [10] in a larger study showing average DON reduction levels of $40 \%$. Moreover, they indicated that the amount of DON retained by cooked pasta consistently decreased by increasing the pasta/water ratio during cooking.
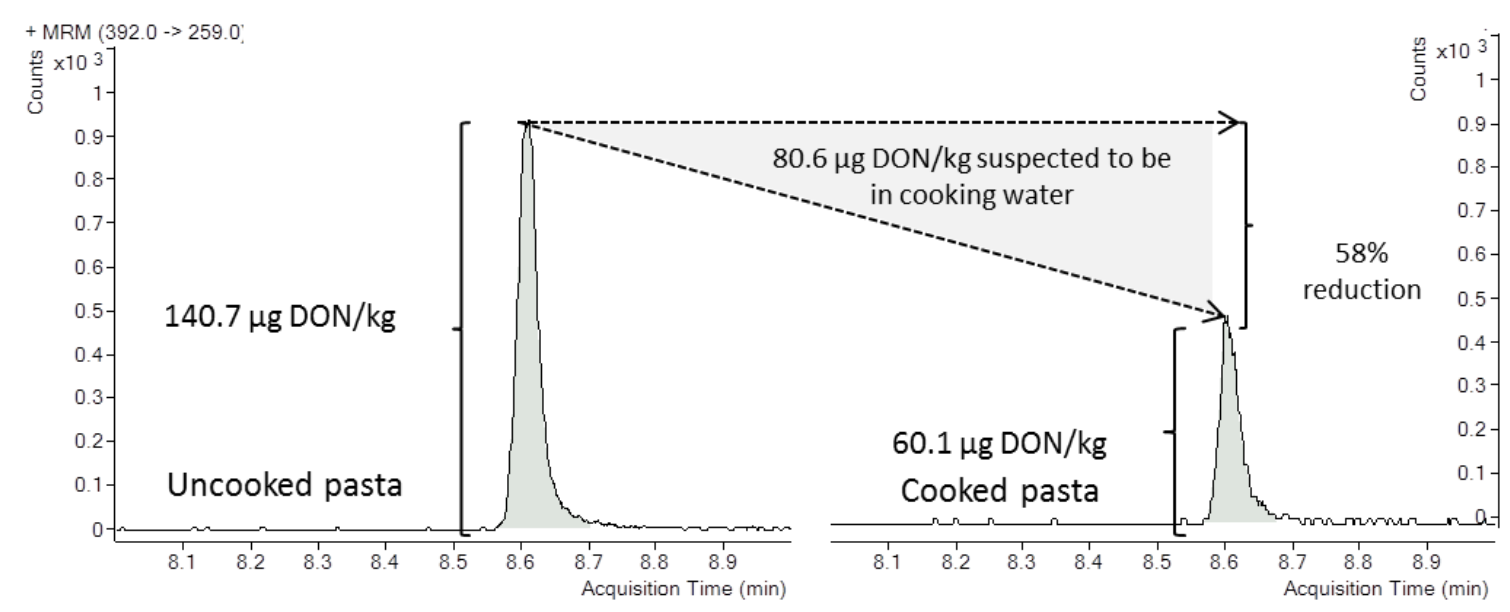

Figure 1. Selected reaction monitoring (SRM) chromatograms of the naturally deoxynivalenol (DON) contaminated pasta before and after culinary treatment at $140.7 \mu \mathrm{g} / \mathrm{kg}$ and $60.1 \mu \mathrm{g} / \mathrm{kg}$, respectively, on a dry weight basis, and percentage of mycotoxin reduction.

\subsection{Deoxynivalenol Content in Food}

All analyzed meals were wheat-based. Within the 15 mycotoxins targeted in the proposed procedure only DON was detected in the analyzed food commodities. This high DON prevalence was highlighted before in cereal and their derivative products by several authors [5] as well as in urine samples [21]. Quantified DON $(n=3)$ in the different food items are presented in Table 2 . The food items presenting the highest concentrations of DON were whole-wheat pasta and toasts with average values of 272.4 and $190.6 \mu \mathrm{g} / \mathrm{kg}$, respectively. Despite beer was the matrix with the lowest DON content, it was the food item with the highest significant contribution to DON intake $(28.6 \mu \mathrm{g})$ because of the consumption data (0.83L). A total DON daily intake throughout the consumption of the followed diet was estimated in 
$49.2 \pm 5.6 \mu \mathrm{g}$. Mean DON contents of the studied food matrices were in line with results reported by other authors [22-25].

Table 2. Overview of contamination level, consumption data and mycotoxin intake contribution of the food items consumed.

\begin{tabular}{ccccc}
\hline $\begin{array}{c}\text { Time of } \\
\text { consumption }\end{array}$ & Food & $\begin{array}{c}\text { Consumption } \\
(\mathbf{g} / \mathbf{d a y})\end{array}$ & $\begin{array}{c}\text { Mean DON } \pm \text { SD } \\
(\boldsymbol{\mu g} / \mathbf{k g})\end{array}$ & $\begin{array}{c}\text { Mean DON intake } \\
(\boldsymbol{\mu g})\end{array}$ \\
\hline $8 \mathrm{am}$ & Toast & 45 & $190.6 \pm 2.3$ & 8.7 \\
$11 \mathrm{am}$ & Breadsticks & 30 & $49.7 \pm 4.6$ & 1.5 \\
$2 \mathrm{pm}$ & Pasta & $67^{\mathrm{a}}$ & $58.2 \pm 2.7$ & 3.9 \\
$7 \mathrm{pm}$ & Wheat beer & 500 & $36.4 \pm 1.8$ & 18.1 \\
$8 \mathrm{pm}$ & Beer & 330 & $32.1 \pm 6.2$ & 10.5 \\
$10 \mathrm{pm}$ & Whole-wheat pasta & $24^{\mathrm{a}}$ & $272.4 \pm 5.9$ & 6.5 \\
& & & $\Sigma$ DON Intake: $49.2 \mu \mathrm{g}$ \\
& Composite $^{\mathrm{b}}$ & 166 & $120 \pm 7.3$ & $19.9 \mu \mathrm{g}$ \\
\hline
\end{tabular}

${ }^{\mathrm{a}}$ on a dry weight basis; ${ }^{\mathrm{b}}$ no beverages included.

The estimated levels of mycotoxins in the composite are also presented in Table 2. DON was quantified at mean level of $120.5 \pm 7.3 \mu \mathrm{g} / \mathrm{Kg}$. A mean DON intake of $19.9 \mu \mathrm{g}$ was obtained throughout the solid food items consumed. The composite's result was in line with the $20.5 \mu \mathrm{g}$ of DON obtained from the sum of each food item. Thus, composite could be presented as an alternative tool to reduce sampling and analysis cost. However, the resulting information concerning contaminants in each selected food item will be missed.

\subsection{DON Content in Urine}

Quantifiable amounts of mycotoxins, and its toxin derivatives that result from its biotransformation, are expected to be found in urine. For instance, DON can be metabolized within the intestinal lumen by gut microbiota, generating the less toxic de-epoxy metabolite known as DOM-1. Further metabolism of DON and ZON to a less toxin metabolite involves the addition of glucuronic acid, catalyzed by UDPglucuronyltransferase [26,27]. As regards mycotoxin conjugation, the uncertainness exists, since it has been related individual difference in the enzymatic system. On the other hand, Turner et al. [28,29] suggested that un-conjugated DON can also persist and it can be excreted in urine. In this line, this work was focused on the investigation of un-metabolized DON as a preliminar step since it needs to be extended in the future to understand the relation between the mycotoxin intake and mycotoxin levels in urine, both metabolized and un-metabolized fractions.

Analyses in urine carried out by other authors have revealed the occurrence of DON in a high incidence of samples. For instance, Gratz et al. [30] reported an incidence of DON in all analyzed samples $(n=54)$. Similarly, DON incidences in urine samples of 33.3\% $(n=27)$ and $67.6 \%(n=34)$ were reported by Rubert et al. [31] and Turner et al. [21], respectively. In this work, a total of $1.87 \mathrm{~L}$ urine was collected as 24-hour urine volume and was in the normal excretion range according to sex and age [32]. DON was quantified at $17.5 \pm 2.7 \mu \mathrm{g} / \mathrm{g}$ creatinine $(n=3)$ (equivalent to $18.8 \pm 3.5 \mu \mathrm{g} / \mathrm{L}$ ). No other mycotoxin was found in the urine sample being according with the results reported by Rubert et al. [31] and Warth et al. [33] who did not find neither any other trichothecenes nor zearalenone 
in the analyzed urine samples. GC-MS/MS chromatograms of naturally DON contaminated composite, beer and urine are shown in Figure 2. Urinary free DON levels of $18.8 \pm 3.5 \mu \mathrm{g} / \mathrm{L}$ (equivalent to $35.2 \pm$ 4.3 $\mu \mathrm{g}$ DON) was calculated in this study. Data found in literature were very similar. For instance, DON average contents of $20.4 \mu \mathrm{g} / \mathrm{L}$ were reported in an Austrian survey [27] ( $n=27$; incidence of $22 \%)$ and a range from 0.5 to $28.8 \mu \mathrm{g} / \mathrm{L}$ were reported in a French study $(n=76$; incidence of $98.7 \%$ ) [26].

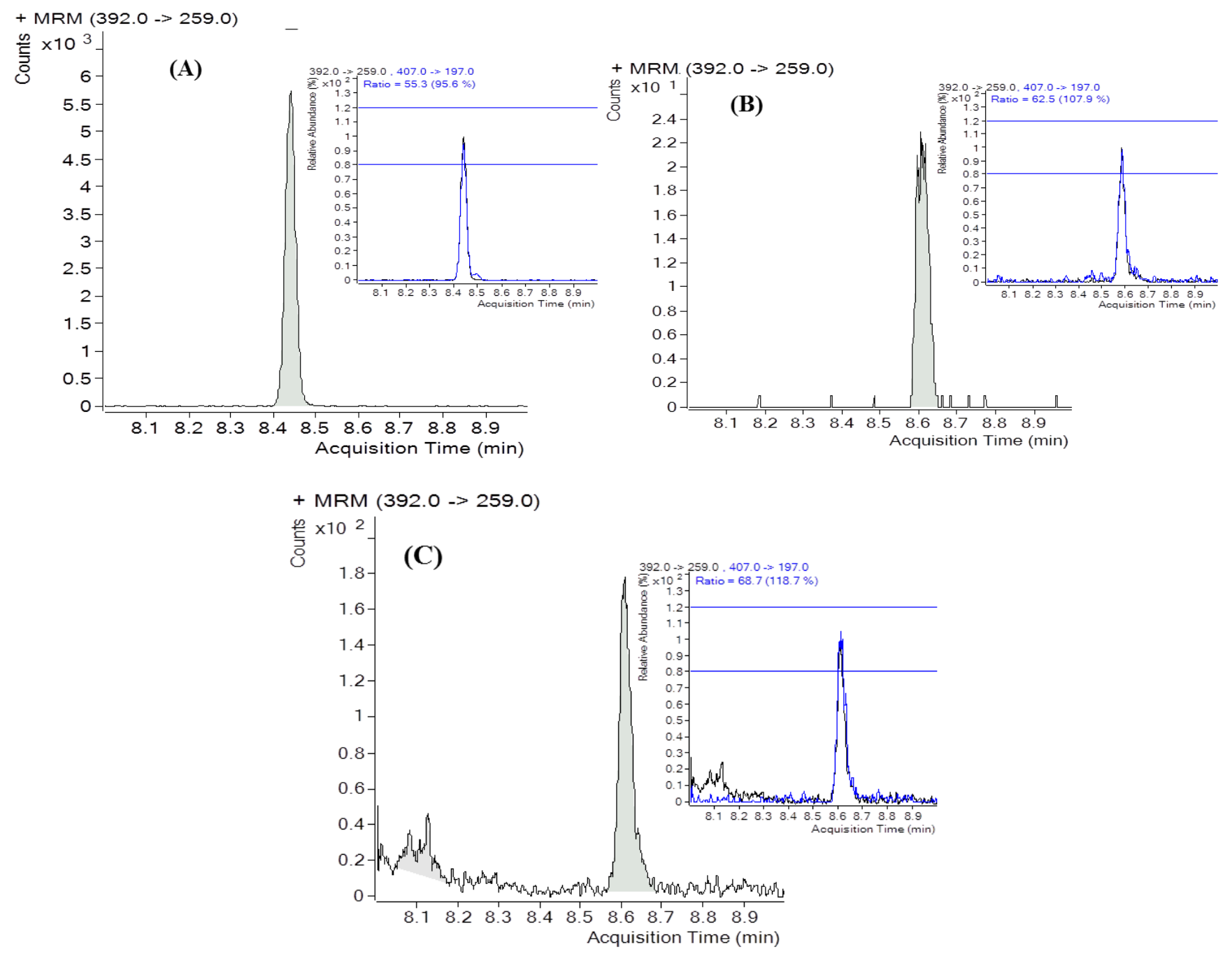

Figure 2. (a) SRM chromatograms of the naturally contaminated composite; (b) wheat-beer and (c) urine by deoxynivalenol at $122.6 \mu \mathrm{g} / \mathrm{kg}, 37.2 \mu \mathrm{g} / \mathrm{kg}$ and $20.8 \mu \mathrm{g} / \mathrm{L}$, respectively.

\subsection{Exposure Estimates}

On the basis of the calculated data, DON daily intake was compared with the established PMTDI of $1 \mu \mathrm{g} / \mathrm{kg}$ bw [13]. The dietary exposure level to DON estimated in the present study was $0.683 \mu \mathrm{g} / \mathrm{kg}$ bw/day, lower than the value set by JECFA. Comparable values as regards dietary exposure to DON were reported in the second French total diet study [34] (mean: $0.379 \mu \mathrm{g} / \mathrm{kg}$ bw/day) as well as in a duplicate diet study carried out in Dutch young children (mean: $0.66 \mu \mathrm{g} / \mathrm{kg}$ bw/day) [35]. These results are also within the latest DON probable daily intake data $(0.34 \mu \mathrm{g} / \mathrm{kg}$ bw/day) reported in the SCOOP 
task 3.2.10 derived from food analysis in Europe [36] and that reported by FAO/WHO $(1.4 \mu \mathrm{g} / \mathrm{kg}$ bw/day) [37].

\section{Experimental Section}

\subsection{Materials}

Mycotoxin standards and metabolites namely de-epoxydeoxynivalenol (DOM-1), DON, 3-acetyldeoxynivalenol (3-ADON), fusarenon-X (FUS-X), diacetoxyscirpenol (DAS), nivalenol (NIV), neosolaniol (NEO), HT-2, T-2, zearalanone (ZAN), $\alpha$-zearalanol ( $\alpha$-ZAL), $\beta$-zearalanol ( $\beta$-ZAL), zearelenone $(\mathrm{ZON}), \alpha$-zearalenol $(\alpha-\mathrm{ZOL}), \beta$-zearalenol $(\beta$-ZOL) were obtained from Sigma-Aldrich (St. Louis, MO, USA). The derivatization reagent composed of BSA (N,O-bis(trimethylsilyl)acetamide) + TMCS (trimethylchlorosilane) + TMSI ( $N$-trimethylsilyimidazole) (3:2:3) was purchased from Supelco (Bellefonte, PA, USA). Sodium dihydrogen phosphate and disodium hydrogen phosphate, used to prepare phosphate buffer, were acquired from Panreac Quimica S.L.U. (Barcelona, Spain).

Certified reference material BRM 003004 (artificially contaminated wheat, DON $1062 \pm 110 \mu \mathrm{g} / \mathrm{kg}$ ) was purchased from Biopure Referenzsubstanzen GmBH (Tulln, Austria).

All solvents, acetonitrile, hexane and methanol (HPLC grade), were purchased from Merck KGaA (Darmstadt, Germany). Anhydrous magnesium sulfate (thin powder) was obtained from Alfa Aesar $\mathrm{GmbH} \& \mathrm{Co}$ (Karlsruhe, Germany); sodium chloride was purchased from Merck and C18-E (50 $\mu \mathrm{m}, 65$ A) was purchased from Phenomenex (Torrance, CA, USA). Picric acid (moistened with water, $\geq 98 \%$ ) and creatinine standard were purchased from Sigma-Aldrich (St. Louis, MO, USA) whereas sodium hydroxide was acquired from BDH Prolabo_-VWR International (Barcelona, Spain).

\subsection{Standard Preparation}

Individual stock solutions of all analytes were prepared at the same concentration $(1000 \mathrm{mg} / \mathrm{L})$ in methanol. The stock solutions were diluted with acetonitrile in order to obtain the appropriate multi-compounds working standard solutions $(50 \mathrm{mg} / \mathrm{L})$. All standards were stored in darkness and kept at $-20{ }^{\circ} \mathrm{C}$ until the GC-MS/MS analysis. Calibration function of both neat solvent standards and spiked samples were established by plotting peak areas versus analyte concentrations in the measured solutions and performing linear regression. Linear range was tested from 0.1 to $100 \mu \mathrm{g} / \mathrm{kg}$ by spiking at eight concentration levels. In order to reveal the presence of matrix effect, matrix-matched calibration prepared by spiking extracts of blank samples with mycotoxins at similar concentrations than the calibration built in neat solvent without any matrix were compared. The slopes of the resulting linear calibration functions were compared and the signal suppression/enhancement due to matrix effects (ME) was determined as follows: $\boldsymbol{M E}(\%)=\frac{\text { Slope }_{\text {matrix-matched calibration }}}{\text { Slope }_{\text {standard in solvent }}} \boldsymbol{x} 100$

\subsection{Study Design and Sampling}

In this $24 \mathrm{~h}$ preliminary study, a farinaceous-based diet was designed and conducted by a 26 year old, healthy male volunteer. Duplicate meals as prepared, served and consumed, based on a "duplicate plate" method were provided for subsequent individual analysis in sterile plastic food 
containers and kept with ice packs in a cooler until they were returned to the laboratory. The selection of food items analyzed in the present study was based on two criteria: first, the food must be identified by GEMS/Food as potential sources of mycotoxins and second, their level of consumption must exceed $1 \mathrm{~g} /$ person day. Four complete meals were consumed during the day of the study as well as two beers in the afternoon. The food groups items selected were bread, wholegrain cereals, pasta, and wheat or barleybased beer. Food items such as pasta (spaghetti) and whole-wheat pasta (little stars) were cooked for 7 min in boiling water. A pasta/water ratio of 1:5 $(\mathrm{w} / \mathrm{v})$ was respected as recommended the preparation mode established in the food packaging labels. Salt and spices were also added as in regular cooking practices.

Upon arrival at the laboratory all meals were weighed and dried in an oven at $100 \pm 4{ }^{\circ} \mathrm{C}$ to reach constant weight for subsequent mycotoxin analysis. The dried products obtained were thoroughly grounded and homogenized using a laboratory mill and kept at $4{ }^{\circ} \mathrm{C}$ under dark and dry conditions into a specific plastic food containers. Note that once dried and milled, all dried products were wheat flour-related foods. For the cooked pasta-based products, both dry cooked form and the dry uncooked ones were subjected to mycotoxin analysis to evaluate the percentage of mycotoxin reduction during the cooking.

A urine sample was collected as $24 \mathrm{~h}$ urine throughout the day of the study, and due to this, it was demonstrated that the main part of absorbed Fusarium toxins showed a rapid elimination within $24 \mathrm{~h}$ after ingestion [38]. Urine collected was stored at $-20{ }^{\circ} \mathrm{C}$ until analysis. The $24 \mathrm{~h}$ period lasted from 8 am to 8 am on the next day to include the first morning urine. A written and approved informed consent was obtained from the volunteer. This project was approved by the University of Valencia Institutional human research Committee and the study purposes and procedures were justified and accepted for this study.

\subsection{Composite Diet Sample}

The composite diet was intended to be representative of $24 \mathrm{~h}$ duplicate diet collected and included all food items consumed over the monitoring period, without beverages. The dried food items were briefly homogenized, carefully mixed, and finally combined keeping the diet proportions.

\subsection{Sample Preparation}

Composite and individual meals were analyzed as described in detail elsewhere [39] in order to know the contribution of each food item. In brief, $5 \mathrm{~g}$ of homogenized sample was added to $25 \mathrm{~mL}$ distilled water and $7.5 \mathrm{~mL}$ of acetonitrile followed by the addition of $4 \mathrm{~g}$ of $\mathrm{MgSO}_{4}$ and $1 \mathrm{~g}$ of $\mathrm{NaCl}$ prior to be shaken vigorously and centrifuged for $3 \mathrm{~min}$ at $4000 \mathrm{rpm}$. Then the upper layer was submitted to a dispersive solid phase extraction (d-SPE) with a mixture of $900 \mathrm{mg}$ of $\mathrm{MgSO}_{4}$ and $300 \mathrm{mg}$ of $\mathrm{C} 18$ and centrifuged for $1 \mathrm{~min}$ at $1500 \mathrm{rpm}$. After centrifugation the liquid extract was separated from the solid salts and finally the extract was evaporated to dryness under nitrogen flow.

Beer samples were first completely degased by sonication for $15 \mathrm{~min}$ prior the analysis. A $10 \mathrm{~mL}$ portion was then used for the analysis. $5 \mathrm{~mL}$ of acetonitrile were added to the sample followed by the addition of the mixture of salts $\left(\mathrm{MgSO}_{4}\right.$ and $\left.\mathrm{NaCl}\right)$ and then submitted to a d-SPE as previously described.

The urine sample was first centrifuged at $4000 \mathrm{rpm}$ for $5 \mathrm{~min}$. A $10 \mathrm{~mL}$ portion of the centrifuged urine was then used for the subsequent analysis as indicated above. 
All dry extracts were added with $50 \mu \mathrm{L}$ of BSA + TMCS + TMSI (3:2:3) and the samples were left for $30 \mathrm{~min}$ at room temperature. The derivatized samples were diluted to $200 \mu \mathrm{L}$ with hexane and mixed thoroughly on a vortex for $30 \mathrm{~s}$. Then the diluted derivatized samples were added with $1 \mathrm{~mL}$ of phosphate buffer (60 mM, pH 7) shaken and the upper layers (hexane phases) were transferred to autosampler vials for the chromatographic analysis.

For quality control, certified reference material BRM 003004 (artificially contaminated wheat, DON $1062 \pm 110 \mu \mathrm{g} / \mathrm{kg}$ ) was used and included. Certified reference material was used as provided without further grinding. It was stored under the same conditions, extracted and determined with the same protocol as the analyzed samples. Each sample was analyzed in triplicate and measured in separate batches.

\subsection{GC-MS/MS Method}

A GC system Agilent 7890A coupled with an Agilent 7000A triple quadrupole mass spectrometer with inert electron-impact ion source and an Agilent 7693 autosampler (Agilent Technologies, Palo Alto, CA, USA) were used for MS/MS analysis. Chromatographic separation was achieved on a HP-5MS $30 \mathrm{~m} \times 0.25 \mathrm{~mm} \times 0.25 \mu \mathrm{m}$ capillary column. One microliter of the final clean extract of mycotoxins was injected in splitless mode (equivalent to $25 \mathrm{mg}$ of dried food matrix) at $250{ }^{\circ} \mathrm{C}$ in programmable temperature vaporization (PTV) inlet employing helium as carrier gas at fixed pressure of 20.3 psi. The oven temperature program was initially $80^{\circ} \mathrm{C}$, and the temperature was increased to 245 ${ }^{\circ} \mathrm{C}$ at $60{ }^{\circ} \mathrm{C} / \mathrm{min}$. After a $3 \mathrm{~min}$ hold time, the temperature was increased to $260{ }^{\circ} \mathrm{C}$ at $3{ }^{\circ} \mathrm{C} / \mathrm{min}$ and finally to $270{ }^{\circ} \mathrm{C}$ at $10{ }^{\circ} \mathrm{C} / \mathrm{min}$ and then held for $10 \mathrm{~min}$. Chromatographic analysis time was performed in $17 \mathrm{~min}$, which reached the requirement for a high throughout determination [39].

The mass spectrometer operated in electron impact ionization (EI, $70 \mathrm{eV}$ ). The source and transfer line temperatures were 230 and $280{ }^{\circ} \mathrm{C}$, respectively. The collision gas for MS/MS experiments was nitrogen, and the helium was used as quenching gas, both at $99.999 \%$ purity supplied by Carburos Metálicos S.L. (Barcelona, Spain). Data was acquired and processed using the Agilent Masshunter version B.04.00 software [39].

\subsection{Calculation of DON Daily Intake}

A deterministic approach was applied for the calculation of DON dietary exposure. The volunteer filled a 1-day food consumption record and was asked to provide his body weight (bw: $72 \mathrm{~kg}$ ). DON daily intake, expressed as $\mu \mathrm{g}$ DON/kg bw, was calculated by combining food consumption ( $g$ foodstuff/kg bw/day) with DON food contamination ( $\mu \mathrm{g}$ DON/g foodstuff) data. The contribution of each food to the average dietary exposure was also calculated.

\subsection{Creatinine Analysis}

Creatinine urinary levels were determined based on a spectrophotometric method slightly modified [40]. In summary, $3.5 \mathrm{mM}$ picric acid was reacted with $1000 \mathrm{mM} \mathrm{NaOH}$ to form alkaline picrate. This solution was stored in the dark in an amber glass recipient. Alkaline picrate $(1 \mathrm{~mL})$ was reacted with $1 \mathrm{~mL}$ of diluted urine $(1 / 10, v / v$, in ultrapure water). The optical density was measured at $500 \mathrm{~nm}$ after 
30 min using a Shimadzu mini 1240 spectrophotometer. Mycotoxin urinary concentrations were correlated to the creatinine content of a sample expressed as $\mu \mathrm{g} / \mathrm{g}$ creatinine.

\section{Conclusions}

A total DON daily intake derived from the $24 \mathrm{~h}$ duplicate diet study amounted to $49.2 \pm 5.6 \mu \mathrm{g}$ whereas $35.2 \pm 4.3 \mu \mathrm{g}$ of DON were quantified in the urine collected in the same. DON incidence in urine of the participant confirms its exposure to DON and evidences the usefulness of DON and its metabolites in urine as a biomarker of exposure to such contaminants. The values of DON PDI estimated herein with the urinary biomarker approach matched quite well the intake derived from food analysis. DON data was further correlated to the established DON PMTDI value in order to obtain a risk characterization approach. DON daily intake represented a $68.3 \%$ of the established PMTDI. The obtained data from this preliminary study is subjected to intra- and inter-day variations. Therefore, this experiment needs to be extended to a larger group of individuals to investigate these variations and to elucidate the relation between ingested mycotoxins and excreted ones and their corresponding metabolites in humans. In this sense, the in vitro digestibility/metabolic models are very useful to complete the full-scale metabolism studies.

\section{Acknowledgments}

This work was supported by the Spanish Ministry of Economy and Competitiveness (AGL2013-43194-P) and Ministry of Education (No. AP2010-2940) F.P.U. Grant.

\section{Author Contributions}

Guillermina Font and Jordi Mañes conceived and designed the experiments; Yelko Rodríguez-Carrasco, Houda Berrada performed the experiments, analyzed the data and wrote the paper.

\section{Conflicts of Interest}

The authors declare no conflict of interest.

\section{References}

1. FAO. Food Balance Sheet. Available online: http://faostat3.fao.org/faostat-gateway/go/to/home/E (accessed on 1 November 2014).

2. Haschek, W.M.; Voss, K.A. Chapter 39-Mycotoxins. In Haschek and Rousseaux's Handbook of Toxicologic Pathology, 3rd ed.; Haschek, W.M., Rousseaux, C.G., Wallig, M.A., Eds.; Academic Press: Boston, MA, USA, 2013; pp. 1187-1258.

3. Alassane-Kpembi, I.; Kolf-Clauw, M.; Gauthier, T.; Abrami, R.; Abiola, F.A.; Oswald, I.P.; Puel, O. New Insights into mycotoxin mixtures: The toxicity of low doses of type B trichothecenes on intestinal epithelial cells is synergistic. Toxicol. Appl. Pharmacol. 2013, 272, 191-198.

4. Antonissen, G.; Martel, A.; Pasmans, F.; Ducatelle, R.; Verbrugghe, E.; Vandenbroucke, V.; Li, S.; Haesebrouck, F.; van Immerseel, F.; Croubels, S. The impact of Fusarium mycotoxins on human and animal host susceptibility to infectious diseases. Toxins 2014, 6, 430-452. 
5. Marin, S.; Ramos, A.J.; Cano-Sancho, G.; Sanchis, V. Mycotoxins: Occurrence, toxicology, and exposure Assessment. Food Chem. Toxicol. 2013, 60, 218-237.

6. Cnudde, F. Safe Foods - Promoting Food Safety through a New Integrated Risk Analysis Approach for Foods. 2005, 30, 194-195.

7. RASFF. Rapid Alert System for Food and Feed. Available online: http://ec.europa.eu/food/safety/ rasff/index_en.htm (accessed on 6 November 2014).

8. Lindblad, M.; Gidlund, A.; Sulyok, M.; Börjesson, T.; Krska, R.; Olsen, M.; Fredlund, E. Deoxynivalenol and other selected Fusarium toxins in swedish wheat-Occurrence and correlation to specific Fusarium species. Int. J. Food Microbiol. 2013, 167, 284-291.

9. Kim, D.; Lee, I.; Do, W.; Nam, W.; Li, H.; Jang, H.; Lee, C. Incidence and levels of deoxynivalenol, fumonisins and zearalenone contaminants in animal feeds used in Korea in 2012. Toxins 2014, 6, $20-32$.

10. Visconti, A.; Haidukowski, E.M.; Pascale, M.; Silvestri, M. Reduction of deoxynivalenol during durum wheat processing and spaghetti cooking. Toxicol. Lett. 2004, 153, 181-189.

11. EC No 1881/2006. Commission Regulation (EC) No 1881/2006 of 19 December 2006 setting maximum levels for certain contaminants in foodstuffs (Text with EEA Relevance).

12. EC No 1126/2007. Commission Regulation (EC) No 1126/2007 of 28 September 2007 amending Regulation (EC) no 1881/2006 setting maximum levels for certain contaminants in foodstuffs as regards Fusarium toxins in maize and maize products (Text with EEA Relevance).

13. Joint FAO/WHO Expert Committee on Food Additives (JECFA). Safety evaluation of certain mycotoxins in food. Food and Agriculture Organization: Rome, Italy, 2001; pp. 281-320.

14. WHO (World Health Organisation). Guidelines for the Study of Dietary Intakes of Chemical Contaminants. World Health Organisation: Geneva, Switzerland, 1985; p. 101.

15. Sakuma, H.; Watanabe, Y.; Furusawa, H.; Yoshinari, T.; Akashi, H.; Kawakami, H.; Saito, S.; Sugita-Konishi, Y. Estimated dietary exposure to mycotoxins after taking into account the cooking of staple foods in Japan. Toxins 2013, 5, 1032-1042.

16. Šarkanj, B.; Warth, B.; Uhlig, S.; Abia, W.A.; Sulyok, M.; Klapec, T.; Krska, R.; Banjari, I. Urinary analysis reveals high deoxynivalenol exposure in pregnant women from Croatia. Food Chem. Toxicol. 2013, 62, 231-237.

17. Solfrizzo, M.; Gambacorta, L.; Visconti, A. Assessment of multi-mycotoxin exposure in Southern Italy by urinary multi-biomarker determination. Toxins 2014, 6, 523-538.

18. Mirocha, C.J.; Pathre, S.V.; Robison, T.S. Comparative metabolism of zearalenone and transmission into bovine milk. Food Cosmet. Toxicol. 1981, 19, 25-30.

19. Warth, B.; Sulyok, M.; Berthiller, F.; Schuhmacher, R; Krska, R. New insights into the human metabolism of the Fusarium mycotoxins deoxynivalenol and zearalenone. Toxicol. Lett. 2013, 220, 88-94.

20. EC No 401/2006. Commission Regulation (EC) No 401/2006 of 23 February 2006 laying down the methods of sampling and analysis for the official control of the levels of mycotoxins in foodstuffs (text with EEA relevance).

21. Turner, P.C.; Hopton, R.P.; White, K.L.; Fisher, J.; Cade, J.E.; Wild, C.P. Assessment of deoxynivalenol metabolite profiles in UK adults. Food Chem. Toxicol. 2011, 49, 132-135. 
22. Edwards, S.G. Fusarium mycotoxin content of UK organic and conventional wheat. Food Addit. Contam. A 2009, 26, 496-506.

23. González-Osnaya, L.; Corteé, C.; Soriano, J.M.; Moltó, J.C.; Mañes, J. Occurrence of deoxynivalenol and T-2 toxin in bread and pasta commercialised in Spain. Food Chem. 2011, 124, $156-161$.

24. Juan, C.; Ritieni, A.; Mañes, J. Occurrence of Fusarium mycotoxins in Italian cereal and cereal products from organic farming. Food Chem. 2013, 141, 1747-1755.

25. Rodríguez-Carrasco, Y.; Moltó, J.C.; Berrada, H.; Mañes, J. A survey of trichothecenes, zearalenone and patulin in milled grain-based products using GC-MS/MS. Food Chem. 2014, 146, 212-219.

26. Turner, P.C.; Hopton, R.P.; Lecluse, Y.; White, K.L.M.; Fisher, J.; Lebailly, P. Determinants of urinary deoxynivalenol and de-epoxy deoxynivalenol in male farmers from Normandy, France. J. Agric. Food Chem. 2010, 58, 5206-5212.

27. Warth, B.; Sulyok, M.; Fruhmann, P.; Berthiller, F.; Schuhmacher, R.; Hametner, C.; Adam, G.; Fröhlich, J.; Krska, R. Assessment of human deoxynivalenol exposure using an LC-MS/MS based biomarker method. Toxicol. Lett. 2012, 211, 85-90.

28. Turner, P.C.; Taylor, E.F.; White, K.L.M.; Cade, J.E.; Wild, C.P. A comparison of 24 h urinary deoxynivalenol with recent v. average cereal consumption for UK adults. Br. J. Nutr. 2009, 102, 1276-1279.

29. Turner, P.C.; White, K.L.M.; Burley, V.J.; Hopton, R.P.; Rajendram, A.; Fisher, J.; Cade, J.E.; Wild, C.P. A comparison of deoxynivalenol intake and urinary deoxynivalenol in UK adults. Biomarkers 2010, 15, 553-562.

30. Gratz, S.W.; Richardson, A.J.; Duncan, G.; Holtrop, G. Annual variation of dietary deoxynivalenol exposure during years of different Fusarium prevalence: A pilot biomonitoring study. Food Addit. Contam. A 2014, 31, 1579-1585.

31. Rubert, J.; Soriano, J.M.; Mañes, J.; Soler, C. Rapid mycotoxin analysis in human urine: A pilot study. Food Chem. Toxicol. 2011, 49, 2299-2304.

32. Schoen, T.; Blum, J.; Paccaud, F.; Burnier, M.; Bochud, M.; Conen, D. Factors associated with 24hour urinary volume: The Swiss salt survey. BMC Nephrology 2013, 14, 246.

33. Warth, B.; Petchkongkaew, A.; Sulyok, M.; Krska, R. Utilising an LC-MS/MS-based multi-biomarker approach to assess mycotoxin exposure in the Bangkok metropolitan area and surrounding provinces. Food Addit. Contam. A 2014, 31, 2040-2046.

34. Sirot, V.; Fremy, J.; Leblanc, J. Dietary exposure to mycotoxins and health risk assessment in the second French Total Diet Study. Food Chem. Toxicol. 2013, 52, 1-11.

35. Schothorst, R.C.; Jekel, A.A.; Van Egmond, H.P.; de Mul, A.; Boon, P.E.; Van Klaveren, J.D. Determination of trichothecenes in duplicate diets of young children by capillary gas chromatography with mass spectrometric detection. Food Addit. Contam. 2005, 22, 48-55.

36. Gareis, M.; Zimmermann, C.; Schothorst, R. Collection of Occurrence Data of Fusarium Toxin in Food and Assessment of Dietary Intake by the Population of EU Member States. Final Report SCOOP Task 2003, 3, 10.

37. FAO/WHO. Safety Evaluation of Certain Mycotoxins in Food; World Health Organisation: Geneva, Switzerland, 2001; pp. 281-387. 
38. Cavret, S.; Lecoeur, S. Fusariotoxin transfer in animal. Food Chem. Toxicol. 2006, 44, 444-453.

39. Rodríguez-Carrasco, Y.; Berrada, H.; Font, G.; Mañes, J. Multi-mycotoxin analysis in wheat semolina using an acetonitrile-based extraction procedure and gas chromatography-tandem mass spectrometry. J. Chromatogr. A 2012, 1270, 28-40.

40. Njumbe Ediage, E.; Diana Di Mavungu, J.; Song, S.; Wu, A.; Van Peteghem, C.; De Saeger, S. A direct assessment of mycotoxin biomarkers in human urine samples by liquid chromatography tandem mass spectrometry. Anal. Chim. Acta 2012, 741, 58-69.

(C) 2015 by the authors; licensee MDPI, Basel, Switzerland. This article is an open access article distributed under the terms and conditions of the Creative Commons Attribution license (http://creativecommons.org/licenses/by/4.0/). 